\title{
MEDICAL INNOVATION AS A PROCESS OF TRANSLATION. A CASE FROM THE FIELD OF TELEMEDICINE
}

\author{
Davide Nicolini ${ }^{1}$ \\ Warwick Business School \\ University of Warwick \\ Coventry, CV4 7AL \\ Fax $+44(0) 2476524656$ \\ Davide.nicolini@wbs.ac.uk
}

\begin{abstract}
The paper makes a case for the use of Sociology of Translation as a way of integrating the classical rational and stepwise view of innovation, showcasing its capacity to produce accounts of innovation that are process oriented, sensitive to contextual conditions, and attentive to its political, conflictual, and institutional aspects. It does so by utilising the approach to study the establishment and mainstreaming of cardiac telecare in Northern Italy.

Building on the results of a three year longitudinal study, the paper describes the process through which this innovative approach carved a space within the existing texture of medical practices by enrolling in successive waves a range of allies and support. The detailed narration brings to the fore some crucial aspects of the local processes of negotiation and struggle, and, more in general, the work and effort that goes into the making of any innovation. The paper concludes that this way of studying and narrating innovation is particularly apt at bringing back time, effort, and politics into the account of the innovation process.
\end{abstract}

\section{KEYWORDS:}

Innovation

Diffusion

Healthcare

Actor Network Theory

Sociology of Translation

Telemedicine

\section{${ }^{1}$ ACKNOWLEDGMENTS}

To appear in British Journal of Management, 21(4). The data discussed in the paper were collected in collaboration with Rino Fasol from the Department of Sociology and Social Research of the University of Trento (Italy). His contribution is gratefully acknowledged. I am also in debt to Jacky Swan, Harry Scarbrough, Bjørn Erik Mørk, and the anonymous reviewers of the journal for their precious comments on previous versions of this manuscript. I also wish to thank Dawn Coton for her invaluable editorial support. Financial support for this research was provided in part by the Provincia Autonoma of Trento (Italy), Progetto Scientifico No. 62001. 


\section{INTRODUCTION: A PROXIMAL APPROACH TO THE STUDY OF INNOVATION IN HEALTHCARE}

For the last two decades a growing number of authors have called for more attention to process, conflict and interests when writing about innovation in organizations (see e.g., Van de Ven and Rogers, 1988; Robertson and Swan, 1996; Van de Ven et al., 1999; Greenhalgh et al., 2004a; Hargrave and Van de Ven, 2006; Swan and Scarbrough, 2005). Although writing from different perspectives, these authors agree about the need to integrate the classical rational and stepwise view of innovation with approaches which are more process oriented, more sensitive to the contextual conditions, and more sensitive to the power, conflictual, and institutional dimension of the phenomenon. The call is thus for interpretive studies which are capable of capturing in detail the social and material process which is associated with the journey of all innovation. Only in this way, it is argued, can we account for the empirical evidence that innovations proceed according to a fuzzy logic, following multiple tracks, proliferating into many ideas, involving a number of people, and, above all, continually mutating in the process.

This appeal, which has been heard throughout the field of innovation studies, has been particularly persistent in the area of healthcare innovation. The argument in this case is that the disregard for interests, conflict, and for the role of institutions in the circulation of innovation is especially consequential in this sector, which is by nature highly professionalised and politically charged (Swan and Newell, 1995; Dopson, 2001; Fitzgerald et al., 2002; Greenhalgh et al., 2004a; Greenhalgh et al., 2004b; Fleuren et al., 2004; Dopson and Fitzgerald, 2005; Dopson, 2005). Ignore these aspects, say the authors, and you are unlikely to understand the process through which medical innovations become taken for granted in the daily activity of health practitioners.

The call appears particularly convincing if we pause to observe that the great majority of studies in this area have been carried out using a distal approach to the study of organizational phenomenal (Cooper and Law, 1995; Greenhalgh et al., 2004a). Cooper and Law (1995) call "distal" the way of studying organizational phenomena which privileges results and outcomes, the "finished" things or objects of thought and action (p. 239). Historically speaking, distal approaches subscribe to a rational and predictive discourse that grants social scientists the role of experts and legislators. Academics are thus expected to contribute to the reduction of complexity and variety through building taxonomies, generating causal models, and developing general propositions, with the (supposed) aim of facilitating the work of decision-makers.

Distal approaches are contrasted to an alternative way of approaching organizational analysis which Cooper and Law (1995) describe as proximal thinking. Proximal thinking views organizations "as mediating networks, as circuits of continuous contact and motion - more like assemblages of organizing" (Cooper and Law, 1995: 239). From a proximal view organizational scholars should not focus on measuring effectiveness and predicting outcomes, so much as describing and clarifying processes, opening "black boxes", and uncovering the mechanisms that sustain what appears and what we experience as ordered configurations and patterned social order. Proximal organizational studies are thus about understanding how and why organizational phenomena and innovations are the way they are, what constellation of interests, desires 
and powers keeps them in place, and, by implication, how things could have been different.

In the area of innovation studies, a proximal approach could be metaphorically reframed as the effort of opening the black box of the S-curve (Coleman et al., 1966; Rogers, 1995; Geroski, 2000). Opening the black box of the S-curve (or any other general model) should not be understood as a way of denying its empirical evidence, so much as zooming-in on the processes that sustain the emergence of this type of macro-trend. In healthcare, for example, this entails generating rich and detailed description of how medical innovations become taken-for-granted aspects of the daily activity of health practitioners, how is it that some of these innovations easily find their ways into hospitals and surgeries, while others remain unused for years, in spite of the huge amounts of money poured into their development and promotion.

This endeavour, however, requires the development of new conceptual frameworks, new ways of doing research and "new vocabularies" (Rorty, 1989). My main aim in this paper will be to demonstrate that the Sociology of Translation constitutes one of these vocabularies $^{2}$. By applying the Sociology of Translation to the emergence and mainstreaming of a new telemedicine practice in northern Italy I hope to show that this approach is particularly suitable for bringing time, effort, and politics back in the study of the emergence, circulation, and institutionalisation of innovations. As it will appear from the case of telemedicine, this way of investigating and describing the innovation process reveals interests and negotiations, bringing fully to the fact that medical innovation is often in the hands of the collective actors involved and largely depends on how well it will serve their local and global interests.

The article is organised as follows. I will start by briefly introducing the Sociology of Translation (SoT) and its contribution to the understanding of innovation and its circulation. After a short introduction to the field of telemedicine, I will then discuss the emergence of tele-cardiology in northern Italy, drawing on the results of a three year longitudinal study. I shall conclude with some considerations on the proactive, interestlead, and material dimension of the circulation of medical innovation and the central role played by institutions in this process.

\section{THE SOCIOLOGY OF TRANSLATION AND THE STUDY OF INNOVATION}

The Sociology of Translation (SoT), also known as Actor Network Theory (ANT), is based on a combination of the idea that actants owe their position and power to the network of characters to which they are related (so that behind any actor there is a network of relationships that speak through him, her, or it) and the related notion that anything put in another place becomes a different thing (Czarniawska and Sevon, 2005). When used in technology and innovation studies, it makes us see that the authorship of innovation rarely lies with individual geniuses or heroic entrepreneurs and is rather to be attributed to a complex array of human and non-human elements that need to be considered as heterogeneous and agential configurations (Dopson, 2005). It also focuses

\footnotetext{
${ }^{2}$ For two recent alternative proposals see, e.g., Dopson (2005) and Hargrave and Van de Veen (2005). The former suggests adopting a configurational approach after the work of Elias; the latter points to social movement theory as a promising new vocabulary for understanding in a processual way the circulation and spread on innovation.
} 
on the fact that the circulation of innovation requires work and energy that can only be provided by the interests of those involved. Finally, it draws attention to the fact that in order to circulate and to succeed, innovative practices and artefacts need to be different things for different people. As such, the circulation of innovation depends on the symbolic, interpretive, or material transformation of the innovation: to circulate and to transfer is to transform.

The SoT uses the idea of translation in a geometric, semiotic, and political sense. First, translation captures the movement of an entity in space and time through which associations and relations are established. Any translation is the result of the active work of heterogeneous mediators which carry meaning and interests. Second, it signals that this movement from one context to another always implies a shift in meaning; the reference here is to semiotics and the inevitable betrayal implicit in translating from one language to another, but also to the fact that this is a way of establishing and cementing relationships. As actors from the outset have a diverse set of interests, the stabilisation of any form of association or relationship rests crucially on the ability to translate, that is, re-interpret, re-present or appropriate, others' interests to one's own through some process of negotiation. Intermediaries and mediators (images, texts and inscriptions, laws and regulations, stories, disciplined bodies, contracts, money) thus act as boundary objects (Star and Greisemer, 1989). Third and final, translation always has a political meaning too, in that establishing and consolidating associations always involves pursuing specific interests, creating differences and sustaining unequal power relationships. Intermediaries, for example, always constitute a tentative form of control, in that they mediate the desires, expectations and control efforts of those who have created them. In so doing they tend to produce reaction, resistance and counterinterpretation. In the world of translation, conflict over meaning and politicking is the name of the game.

When applied to the study of the circulation of ideas and practices, the idea of translation suggests that the so called diffusion of transfer of innovation is better understood as a process whereby ideas and practices are disembedded from their existing context and translated in a different place or time. This in turn implies that:

- The travel of ideas can be respecified in term of a loose three steps process (Czarniawska and Joerges, 1995; Czarniawska and Sevon, 1996; 2005). For an innovative idea to circulate it must first be disembedded and materialised into an intermediary, that is, it must be separated from its original context and translated into an object such as a text, a representation, or a prototype. Second, the ideaincarnated-in-an-object travels through time and space until, third, it is re-translated in some other locale in view of the new contextual conditions and existing practices. Finally, the idea is institutionalised and eventually taken for granted - its origins carefully concealed from view.

- New ideas and practices do not impose themselves on the new adopter thanks to their supposed inherent innovativeness. In fact, at any point in time, there will always be several new ideas in circulation. Accordingly, disseminating or broadcasting an idea is not enough, and the impetus for imitation must come from the imitators themselves (Czarniawska and Sevon, 1996).

- The fate of the new ideas lies very much in the hand of the receivers each of whom may react to it in different ways. They may accept it, modify it, deflect it, 
betray it, add to it, appropriate it, or let it drop. It follows that translation processes are always anchored in local contexts: the new ideas have to resonate with local interests in order to be taken up, and the way in which they will be attributed meaning will be heavily dependent on the local existing conditions.

- Translation proceeds along uninterrupted chains: if you want an idea or practice to move in time and space you need to find new sources of energy (Latour, 2005).

At each step, the idea or practice is likely to be somewhat reinterpreted and modified in order to fit the interests of the new imitators. The process of translation is thus always open-ended and uncertain and instead of a process of transmission we have a process of continuous transformation.

- An idea or practice becomes the object of imitation if it is taken up by an overwhelming number of people or organisations (Fujimura, 1995). The circulation of innovation, from some point on, depends on the generation of a robust bandwagon. The emergence of a bandwagon can be either fortuitous or the result of intentional effort. More often, a bandwagon emerges from a combination of the two. In all cases, the creation of a bandwagon depends largely on the circulation of suitable intermediaries that can enrol new powerful allies and build a network of relationships and dependencies. When such a network grows enough, it cannot be ignored and it automatically becomes an object of imitation and later a source of conformist pressure.

- The creation of a bandwagon requires some manoeuvres of exclusion as well as a trial and error process of negotiation, by which all entities find a place in the emerging configuration. In this sense, the translation of ideas and practices is necessarily also a process of empowerment and disempowerment, in which some way of understanding and defining the word prevails over others. Translating is therefore a form of power struggle in which the innovation is both the arena and what is to be sized (Czarniawska and Hernes, 2005).

The approach thus puts the attention on what circulates among actants (intermediaries), on how battles over meaning and over the shape of existing institutional arrangements are fought (e.g., what constitutes a 'diagnosis'), on the techniques used for making proselytes, on the process through which the ideas are translated locally, and on the effort to give the new way of doing things the necessary legitimization. It emphasises that the innovation itself is both the object and the arena of all these political and negotiations processes, so that to transfer is necessarily to transform.

In the next section I will use this conceptual toolbox to examine the emergence and 'spread' of a particular type of telemedicine, that is, telemonitoring serious chronic heart failure patients. My main aim will be to illustrate how through the use of these categories it is possible to produce a proximal description of the circulation and stabilisation of medical innovation which emphasizes its processual, networked, and interests lead nature.

The data are derived from a three year longitudinal research conducted by the author in northern Italy. The research included several periods of participant observation in two specialised medical centres and in a telemedicine call centre; a number of ethnographical and semi-structured interviews with doctors, nurses, managers, and health officials; the attendance at meetings, promotional workshops, and training sessions; the examination of technical and policy documents, reports, and scientific 
materials. The research was part of a larger project on the social and organisational implications of telemedicine. The project is described in detail in Fasol and Nicolini (2004) and Nicolini (2006; 2007 $\left.{ }^{3}\right)$.

\section{THE CASE OF TELEMONITORING}

\subsection{Telemedicine, telecare and telemonitoring}

The term "telemedicine" is commonly employed to indicate the use of communication and information technologies in the context of health care activities. An increasingly important sub-set of telemedicine is telecare. Barlow et al (2006) define telecare as a set of services bringing care directly to the users (p.397). It includes the provision of health and service information (e.g., health advice, access to self-help groups), safety and security monitoring (e.g., monitoring of critical aspects of house holding such as gas let on), and personal telemonitoring (e.g., the telemonitoring of vital parameters such as breathing and heartbeat and changes in life style). According to Barlow et al. (2006) personal telemonitoring ${ }^{4}$ critically differs from other types of telemedicine (such as, e.g., teleradiology or teledermatology), in that it is mainly aimed at crisis response and prevention. As such, it is particularly appealing in all those conditions which require long term care and that until recently required long and expensive periods of hospitalisation.

\subsection{What is chronic heart failure?}

Serious chronic heart failure (CHF) is a highly debilitating chronic condition affecting a growing number of patients, most of them aged 60 and over. The pathology afflicts about $1-3 \%$ of the population, but in the over 75 age group this rises to $10 \%$ (McMurray and Stewart, 2003). In very simple terms, heart failure means that the heart doesn't work properly. Acute crises are not uncommon and when they occur, patients need to be rushed to the hospital, put under intensive care, and "stabilized" with an appropriate cocktail of medicines. Until a few years ago this condition was typically treated through a recurrent pattern of hospitalization, intensive therapy, discharge, deterioration of condition and subsequent new hospitalization. More recently, however, this cycle, however, contrasted with the emerging evidence that unless the disease was properly managed, patients' conditions could seriously deteriorate over time. In this context, health practitioners in different parts of the world started to consider the possibility of using information and communication technologies, i.e., telemedicine, for addressing the issue in a novel way. In Italy, this idea first took root in Lombardy. One of the first sites to experiment with this new approach was the centre of G. a branch of a national medical foundation with several outlets in different cities which specialized in the care of chronic heart conditions.

\subsection{Telemonitoring: mythical birth or birth by translation?}

This is how the history of telemonitoring was told to me by S., the telemedicine "champion" at G.:

\footnotetext{
${ }^{3}$ For reason of space, readers are referred to these sources for further details on the research project and methods.

${ }^{4}$ In the rest of the paper I will use the term telemonitoring to refer to "personal telemonitoring" as defined here.
} 
"So I have to tell you the 'history' of telemedicine...well, I was travelling to Canada with the director of my medical centre for a congress...during the trip I started walking down the plane and saw someone reading the conference programme...so I asked him in English: "Ah, are you going to the so and so world conference?" He replied, in English "Yes, where are you from?" he was from a hospital twenty miles from ours. That's how we met and he told us about his experiences with telemedicine... and it all started there...can you imagine it?"

Myth is a superb tool for making what is contingent and fortuitous look like a necessity of history and destiny. Mythical accounts, however, also hide from view the more prosaic reality that building empires and, in our case, assembling telemedicine at G. involved from the beginning a much more mundane process of establishing associations and aligning interests. Telemonitoring in G., emerged thus from the encounter/crossing of a number of practices and existing concerns within a precise landscape of interests.

\subsubsection{A landscape of interests}

Firstly, one should consider that following patients at a distance was already an established habit at G. Nurses would regularly receive calls from patients who asked all sort of questions and provided phone support to the patients even when these had left the ward. Although very much appreciated by the patients, however, this work was not acknowledged and hence was perceived as a burden by the centre personnel.

Second, the medical literature had already provided rather persuasive data which indicated that in the management of CHF patients, frequent contacts were a critical variable for the prevention of heart failure crises. This $\mathrm{c}$ indicated the need for devising novel ways of increasing the level of interaction with the patients without increasing the associated costs.

Third, a current concern was the innovative tradition of the Foundation and expectations that the centre in G. would continue to publish scientific research and appear as a cardiology innovation centre. On this depended, amongst other things, the personal careers of both the director of the centre and Doctor S., later to become the champion of telemedicine. As a leading consultant she was constantly in search of something new. As we have seen above, this something new was just around the corner. To understand what was around the corner, however, we need to step a bit back in time.

\subsubsection{More interests}

In 1997 doctor F. was a cardiology consultant working in a small hospital in a mountain area of northern Italy a few miles from G. In that year he decided to set up a small centre for cardiology tele-consultation in his ward. This tele-consultation was a service for family doctors in the area with the objective of preventing often unnecessary journeys by elderly patients from the mountains to the hospital for check-ups. The new system enabled the family doctors to take an electrocardiogram (ECG) at the patient's home using a portable recorder, send it to the hospital through the normal phone line, and have it interpreted at a distance by Doctor F. or one of his junior cardiologists. If all was well, the arduous and pointless journey through the mountains "for a check-up" could be avoided. Being a fairly junior consultant, doctor F. had to face the resistance of 
his colleagues, who saw his entrepreneurship both as a waste of time and as a threat. He also had to struggle to find the resources for the necessary equipment and the space in the ward. In 1997, he had few allies for fending off these contrary forces: the bandwagon of telemedicine and telecare, in Italy, had not yet started; clinical evidence was still scarce; there were other centres in the countries which already provided this type of service. The odds were stacked against him. As it happened in many entrepreneurial stories, a chance event made the difference between success and failure. During a "live" consultation with a family doctor who was called at a young patient's home in a remote place, doctor F. detected the early stages of a devastating heart attack. A helicopter was sent, the patient was saved, and the event was reported in the papers. Tele-cardiology became an instant local celebrity.

While the short term survival of the project was ensured, it soon became clear that telemedicine in such a small hospital would be limited by the lack of staff and the work overload of the cardiologists and the secretary providing the service. Unwilling to see his hard work go to waste, Dr. F. decided to take a leave of absence from the hospital and set up a telemedicine business. He founded a small company, Telemed ${ }^{5}$, which continued the work he had begun. The initial financial resources for this endeavour were provided by the company producing the gadget for transmitting the ECG over the phone. Further funding came from a large pharmaceutical company which agreed to sponsor the project offering the "ECG over the phone" service as a free promotional benefit for family doctors. For the producer of portable ECG recorders, this was an opportunity to penetrate the new and potentially vast Italian market. For the pharmaceutical company, this was a chance to improve its image.

Doctor F., however, had to overcome a further obstacle. The model of telemedicine he "sold" to his initial client base included the possibility of the family doctor speaking with a consultant cardiologist. While finding cardiologists at hand was somewhat easy while he was operating from within a hospital ward, he had to find an alternative solution now that he was an independent. He started with his local colleagues, who in this were turned from potential obstacles into allies. Finding and convincing a sufficient number of cardiologists, however, was not easy. For the telemonitoring "system" to work, he needed to enrol new allies. It is at this point that the cardiologist-businessman met his colleagues from G. (the mythical encounter described above) and offered them the opportunity of working for him in the telemedicine service for payment. The Foundation, staffed with very experienced cardiologists, was in fact a great source of manpower for the increasing needs of his tele-cardiology centre. Several day shifts, and all the night calls, would be handled by the centre of G., providing local doctors with some welcome extra income.

As all good entrepreneurs, however, Doctor F. foresaw other benefits in associating himself with the centre of G. First, Telemed was a small company and operated in a field whose validity still had to be proven and whose legitimacy was constantly being challenged. In Italy at the end of the 90 s, telemedicine had still a poor reputation in the medical world. Involving the Foundation in the project meant enrolling a powerful legitimate ally capable of covering this side of its construction. This in turn played straight into the interests of the doctor-researchers at G., who could use the data derived

\footnotetext{
${ }^{5}$ The name of the company has been changed to preserve anonimity.
} 
from the telemedicine activities of Telemed (which by that stage had been going on for two years), as a potential data base for their own research.

Second, to ensure the financial viability of the new centre's usage rate, the productivity levels of the equipment and staff had to be high. As we have seen, Doctor F.'s model of telecare was based on the live and immediate contact between a family doctor and a specialised cardiologist. While this model set his approach and centre apart from other companies which were already providing off line readings of ECG sent by phone, it required a specialised centre manned on a $24 / 7$ basis which was very expensive to run. The centre was necessary for relaying the calls between the doctors and the next available cardiologist, decodifying the incoming ECG sent over the phone (a special software was necessary), and forwarding the actual readable test by fax to the cardiologist. It also registered and recorded the cardiologist's opinion providing the caller with a written copy. Such a centre, however, had some high investment and operating costs and needed to work at or near capacity to be viable and profitable. Hence, other "telemedicines" were needed which could use the centre in addition to interpreting distance ECG.

\subsubsection{Telemonitoring: aligning an heterogeneous landscape}

Telemonitoring, or, more precisely, the idea of telemonitoring, emerged thus as the boundary object which could translate and align all these interests. Telemonitoring as a way of handling CHF was not unknown. Mention of similar experiences in the English speaking countries had been published in scientific journals in the sector and they were well known in Italy (Scalvini and Giordano, 2002). Although not unknown, however, telemonitoring was still waiting to be translated in practice in such a way that it could bring all these interests together and make them work as well oiled machine. This, however, required a patient and challenging bricolage work of assembling and aligning all these elements and many others which had remained in the background until then. As noted by many authors (see, e.g., Barlow et al, 2003; 2006) telemonitoring and telecare services are a very complex innovation in that they impinge on a variety of other services and practices. This high level of interdependency and "entrenchment", however, only becomes visible when the idea is translated in practice - hence the very high level of failure of this type of initiative (May and Ellis, 2001).

One of the first acts of alignment was the establishment of a scientific partnership (later to become also a financial cooperation) between the centres of G. and Doctor F.'s company. The scientific partnership was one way of cementing the relationship and distributing roles. The collaboration protocol clearly establishes, in fact, that while data are to be shared, all activities must use the call centre.

The partners in turn created a local space where all the other elements could be aligned: a project. Projects are very efficient translation machines aimed at the alignment of human and non human elements, and this was no exception. Through the project the emerging practice of telemedicine set out to enrol the relevant stakeholder to find a suitable role and play it in a consistent manner (what Callon (1986) calls interessement).

Telemonitoring was thus further adapted or more precisely re-translated so that it could persuade and seduce a variety of actors. For nurses, it became an opportunity for 
fostering their professional identity. For doctors in the ward this was a relief in that nurses would shield them from telephone calls and questions from anxious patients and worried families. It would also reduce the pressure deriving from emergency admissions. For patients, telemonitoring meant much less travel back and forth to the hospital and the reassurance of ready at hand professional advice just one call away. Family doctors saw this as a great improvement in the care of their patients, and the opportunities to delegate the management of these difficult clients to a national centre of excellence. As I have discussed elsewhere (Nicolini, 2007), the attempt at aligning all these elements and assigning them specific roles was anything but a smooth and painless affair.

Expanding medical work in time and space required the creation of new routines (e.g., how to establish the optimal dosage of medicines at a distance) and a redistribution of work which partly collided with the existing work practices (Lehoux et al., 2002). For example, the new arrangement was pushing the existing job description to the limit and the nurses had to develop new ways of making themselves accountable (Nicolini, 2007). They also realised that they were not up to speed with the medicines they were monitoring, and needed to learn new skills and information. Family doctors, on the other hand, were at odds with discussing clinical matters with nurses, and wanted to talk to the doctors (although this defeated the original purpose of the service). Finally, patients too had to take up some of the work for example by responding to the phone, operating the portable ECG devices, carrying out some of the tests that in the hospitals others would do for them, providing the correct data, and following the advice given to them by the nurses. This required them to be much more proactive than they would be in the hospital, where many of these things would have been done for them. As nurses soon discovered, it was not only they who needed some convincing, patients had to be taught how to become tele-patients.

The non human elements collaborated only in part. The portable ECG showed a distinctive tendency to send bad signals, and the nurses had to teach the patients how to "hear a good ECG" in order not to have to deal with unusable data. The hospital phone lines were revealed to be old and incapable of supporting the direct link with the Call centre. The nurses had to learn quickly how to diagnose technical faults switching to their back up system when needed (they used an old fax for receiving ECGs from the call centre).

Through a complex activity of "heterogeneity engineering" (Law, 1992), all these elements slowly fell into place. In a very concrete way, the practical meaning of the idea of "telemonitoring" had to be discovered through the negotiation and reciprocal accommodation of all the different elements that needed to work together, from the nurses to the telematic infrastructure. After a few months, this bricolaging process got to the point when the first patients could be enrolled. Patients were given a portable ECG recorder and were trained on how to use it to send their data over the phone using the telemedicine centre described above. At regular intervals specialized nurses contacted the patients, checked the ECG and other parameters. During the call the nurses would also adjust the dosage of medicines if necessary. Only when diagnostic parameters suggest a serious deterioration are medical doctors involved. Otherwise the specialized nurses take care of everything (this happens in about $90 \%$ of cases). The model for the 
service is the nursing triage practice, which is commonly used in Anglo-Saxon countries but still rare in Italy.

The alliance between all these elements appeared to hold. The set of heterogeneous elements that went under the collective name of telemonitoring had begun to inscribe the world.

\subsection{A ruler over a new realm?}

The story of telemonitoring could have stopped here. Monitoring CHF patients at a distance would have become a local social practice, entrenched in the ambit of the many activities of the hospital at G., and used by local health practitioners. This way of handling CHF patients would have been learnt by trainees as part of socialization and would have represented one of the distinguishing characteristics of the local organizational culture of $\mathrm{G}$. In the Italian health care system (and elsewhere) a great many practices end up like this: they survive locally and never become "diffused" because they are supported by a local configuration of interests and resources. Each of these configurations produces a local "compulsory point of passage", usually represented by the translation's sponsor who in this manner earns its profits. The new practice increases its power in her/his little realm. This explains why many innovations remain rooted in the site of their emergence, they never go around, and they disappear/dissolve with the conditions that made them possible or necessary.

However, as we have seen, the interests motivating the birth of telemonitoring were translocal right from the beginning. The legitimacy provided by the institutional visibility of G. was of itself not sufficient to support doctor F.'s and Telemed's attempt to become a compulsory point of passage for a sufficient number of ECGs and phone calls. Nor was it enough for the management of the centre at G., which had more ambitious objectives and saw its centre as a compulsory point of passage for anyone in Italy who was interested in tele-cardiology. A new translation was needed to significantly widen the network of associations defined by the practice of telemonitoring. The objective this time was to make telemedicine at G. appealing for the regional or even national medical community - a move which would create a new realm, at whose head would be telemedicine as practiced at G. and all its components, both human and non human.

This second translation needed thus to engage with science and with scientific institutions which represent a fundamental resource for the translation of medical innovations. Enrolling the scientific institutions would in fact obtain the result of entrusting the emerging practice with a great power-symbolic superiority. In other words, to extend its range of action, telemonitoring had to enrol the entire modern medical disciplinary field of cardiology and present itself "as if" it spoke in its name. For this to happen, however, it was not necessary to enrol all the elements that make up this enormous galaxy. Each institution must have at its disposal a variety of tools so that the interests it represents can be translated rapidly, legitimating and translating part of its authority to the new practice, technology, or drug.

In Western medicine, this mechanism is represented by the scientific experimentation process. A medical practice or therapy that in a randomized control trial (Sackett et al., 
2000) has demonstrated its high efficacy and statistical significance, and that manages to get an important professional body to accept these results, quickly becomes a legitimated way of problematising a particular type of concern. From this moment on, to suggest that telemedicine is not a good way to care for CHF patients, would mean taking on not only the centre at G., as in the initial phase, but also the methods, procedures, and overall logic used by the medical profession to construe their facts.

The mobilisation of scientific institutions thus required a new translation and the production of suitable intermediaries in the form of scientific data and papers. In the spring of 2000 the G. and B. coalition submitted a project for "the definition of a protocol for the telemonitoring of CHF patients" to the Ministry of Public Health. The project aimed at making telemedicine at G, "the" most scientifically advanced method to manage CHF patients. This implied the elimination of other possible systems, for example, old style hospital admissions, by presenting them as demonstrably less effective. The machinery of science had thus been enlisted openly as an instrument of politics and marketing (Law, 1986).

Starting from mid 2001 and for two more years the centre at G. collected data to prove the clinical effectiveness and the satisfaction of the patients and to test the economic viability of the model. The experiment was strategically framed as a comparison between telemonitoring and the traditional way of handling CHF patients so that the latter could be demonstrated to be superior to the former. The data emerging were in turn used to support the problematisation of CHF in terms of telemonitoring, and to position the centre at G. and Telemed as compulsory points of passage. People at G., were in fact not only excellent heterogeneous engineers, but also very good doctorsociologists. Knowing that mobilising science was critical for the success of telemonitoring (and, for their own success), they literally set up a scientific and media campaign. Several articles were submitted and accepted at international conferences and subsequently appeared in refereed journals (we published "as much as we can", as the head of G. put it); popularising articles were published in the local and national press which was invited to the Centre; last but not least, the doctor turned sociologists enrolled other scientific institutions such as the University to which the author belonged. The latter involved a politely concealed ruse: they allowed some researchers (I and my colleagues) to study their centre with no strings attached. One day, they asked if they could mention our "well known university" as a "scientific partner" in their papers and on their site. This was a request that we could not refuse. We had been translated too!

The growing scientific support for telemonitoring was also turned into an opportunity for extending the net of those using the emergent model of telemonitoring and for positioning the centre of $\mathrm{G}$. as the leading national centre for telemonitoring, that is, stating in symbolic terms "telemonitoring are us". This was ensured by organizing a series of presentations at conferences, seminars, and meetings where the provisional data of the research, plus the results of previous researches, were presented.

Other centres of the Foundation and hospitals from other regions decided to start telemedicine services. The centre at G. offered them a complete "telemonitoring translation kit", which included training support needed and access to the Telemed infrastructure for the transit of the ECGs. Slowly but gradually, the action-net 
assembled around the new practice began to expand, capturing more and more elements, increasing the traffic in doctor B.'s call centre (which in the meantime had been expanded and completely brought on line), and increasing the reputation of the Centre of $\mathrm{G}$.

Each of these new sites constituted a further chain of translation that took telemonitoring to a new place. Of course, they all had to go through a localisation process not dissimilar to that described above. The "intermediating kit" sent out by the centre of G. (a one day workshop, a set of slides, some documents) needed in fact to be translated and aligned with the local interests, problems, and material conditions. The growing assemblage, the overarching emerging practice of telemonitoring, was not a monolithic or homogenous entity, as much as a tessellation of local instances (local associations) actively kept together by the new 'broader' discursive and material manoeuvres in the global network.

In sum, the second translation was aimed at reinforcing and promoting telemonitoring by increasing its perceived legitimacy. This was achieved through enlisting science as an ally. Science helped to make the potential of telemonitoring to solve the CHF problem a fact, thus turning telemonitoring into a practice that required less justification and hence less local effort during the translation process. Science, of course, is far from a single monolithic body that can mobilised at will. As I will show in a moment, the scientific world is in itself an arena where controversies and translation battles are fought. However, the enrolment of science was a critical move that allowed telemonitoring to become backed by scientific evidence and by the galaxy of interest and practices that produce it. In this case, then, evidence was not a cause of change as much as a resource that was skilfully deployed within a sophisticated strategy for circulating the innovation. The machinery of science, moreover, also helped to create a discourse of telemonitoring, a way of talking, categorising, and distinguishing which helped the many local instances of translation to start acting as one. The translation not only allowed telemonitoring to be in many places at once, it also allowed all these places to speak with one recognizable voice, that of the centre of G. in other words, with the help of scientific evidence the idea of telemonitoring started to be turned into a wave that the people at G. intended to ride as far as it would get them.

\subsection{The standardization and institutionalization of telemonitoring}

At the end of 2003 telemedicine at G. had already come a long way. The practice had put down deep roots at G., it was being used by a number of centres and hospitals in northern Italy. Many of these sites adopted the standardized Telemed service package, thereby reinforcing the original format and the associated socio-material assemblage. Articles were appearing in specialist journals and the benefits of telemonitoring were discussed on the local and national press. Telemonitoring was even proposed as a topic for the forthcoming national cardiology conferences. Members of the consortium were regularly invited as "experts" in seminars and national commissions on telemedicine.

Despite these early successes, however, a series of factors risked unravelling the complex network that supported telemedicine at G. and its attempt to become the "ruler of the (new) realm of monitoring CHF patients at a distance". 
In the first place, with the increased general interest for telemedicine the G. sociotechnical alliance started to feel the pressure of competition as other centres tried to impose themselves as compulsory centres of passage.

Second, lack of resources threatened to unravel the social-technical relationships upon which telemonitoring was built. With the termination of the ministerial project in 2003, funding to continue the experiment and extend the weight of the practice to the rest of the scientific community was stopped. This lack of resources was critical because the practice and its network of tele-nurses, computers, call centre, etc. demanded a substantial amount of work and resources to produce effects. While the data collected during the research indicated that the new way of managing CHF patients was more economical than the alternatives, this money could not be recovered, given that this new practice had still not been officially endorsed by the healthcare service. The service was saving money, but telemonitoring was starving. Out-of-region patients were asked to pay for the service. Some accepted, others did not. Instead of becoming larger, the network that telemedicine at G. sought to use to envelop the CHF world, began to shrink.

It clearly emerged at $\mathrm{G}$. that the survival and further growth of telemonitoring required further translation which could no longer rely solely on the involvement of the scientific institution, which could provide legitimacy but not resources. The public health system ${ }^{6}$ had to be translated into telemedicine to support the problematisation of CHF in telemedicine terms, not only from the point of view of scientific legitimacy but economic necessity as well. If the move succeeded, telemedicine would become a treatment recognized by the public system, which could fund the service offered by telemedicine as an alternative to traditional ones. In fact, this would transform "telemedicine at G." into the "telemedicine of the "Lombardy Region" (where G. is located), thereby institutionalizing it.

\subsubsection{A new heterogeneous landscape}

As in the local case described above, this new, much wider context was equally populated by a variety of seemingly unconnected interests besides those of the people from $\mathrm{G}$.

First of all, since the first translation of telemonitoring a number of other telemedicine and telecare projects had been put in place including an initial national programme for telemedicine (Ricci, 2002). The momentum of telemedicine and telecare had picked up and could not be ignored especially in regions such as Lombardy, which prides itself for being a beacon in the country.

Second, the Lombardy region, now ruled by a conservative coalition, was interested in pursuing a policy of vigorous cost reductions and the increased involvement of private companies in the provision of healthcare services.

\footnotetext{
${ }^{6}$ In Italy there is a national public health system which is managed and financed by regional governments. The centre at G. and the others are in the Lombardy Region, which is the largest and wealthiest of the Italian regions.
} 
Third, there were a number of hospitals where people had local interests for becoming champions of a new technology. This included not only curious or ambitious clinicians but also managers who were very open to the idea of having "something that would allow us to go in the press with good news for once", as one of my informants put it.

Telemonitoring, this time reinforced by the support of scientific evidence, promised to align all these interests. This third wave of translation and the enrolment of the public system, however, required that the practice be normalised and standardized in order to be costed and controlled. Boundaries and definitions had to be drawn around the practice in order to render its local instances comparable. A new type of intermediary promised to keep the new set of interests together: a standard procedure (that in healthcare is often described as "protocol" or "service model").

Protocols and service models, like standard procedures, plans, edicts, and regulations, include programs of action for the users. They define roles to be played and make implicit or explicit assumptions about what competencies are required, who is going to do what, and how much resource will go into it. The development and piloting of the new service model was of course a favourable opportunity for the centre of G. It allowed the centre to support its telemonitoring activity for another three years, at the end of which the new reimbursement regime was likely to take effect. There was, however, a serious problem. In Lombardy there were other "scientifically legitimated" ways of doing telemonitoring which were capable of translating the existing landscape of interests. The practical meaning of telemonitoring developed at G. had to be partially renegotiated, which, from the point of view of its creators, constituted not a translation as much as a distortion. In effect, the new translation required undoing and redoing some of the work carried out in previous years.

The reason was that the project included two other institutions in addition to G. One in particular, clinic Z, which is a cardiology centre that is famous nationwide. For years this centre used telemedicine practices to look after its recently discharged patients following open heart surgery. Although they had never done telemonitoring for CHF, they developed their own practice and asked for elements from it to be included in the procedure. The practice established at $Z$ applied a form of "hybrid" telemonitoring, partly face to face and partly distant. From its inception, the clinic at Z, unlike G, sent nurses for home calls at set times. The clinic thus aligned similar elements to G. in a different way. The result was to have nurses who were experts at handling patients face to face but not over the phone, and infrastructures capable of reacting to an emergency but not supporting daily supervision like G. The differences between the two approaches produced a strong clash, to the point of stalemate, which slowed down the process for many months. According to accounts by the participants, the conflict initially emerged over the scientific plan. The two centres had both produced scientific literature justifying their respective models and intended to defend them. Since both centres were well known nationally, the dispute on a scientific level could not be resolved. Only later did the economic motivations behind the disagreement emerge. The two different styles of organization had been conceived locally to make the best use of economic resources. For both contestants, the definition of a protocol that laid down programs for action which were different from existing ones meant investing resources to realign their local network and excluding already available resources which would have had to be replaced with others at a higher cost. For example, for G. to accept the 
model at $\mathrm{Z}$ would have meant doing without the services provided by the Telemed call centre and paying for new nurses to do house calls. This move to economic motivations in the discussion helped open the way towards a compromise. Interestingly enough, the compromise was written into the new clinical procedure, which made up the standardized packet for telemedicine. To do this the procedure adopted a resource that appeared to be central to the translation process: ambiguity. The procedure was simply left open: the nurses could go and visit patients or use the phone instead depending on necessity (the expression used was: "on demand and upon careful consideration"). The ambiguity and flexibility thus allowed the procedure to represent the networks that had created it while permitting others to adopt translated telemonitoring in practice.

The translation had some further relevant implications for the practice of telemonitoring. For example, legal issues required a more precise division of labour between normal and tutor tele-nurse. The more serious CHF cases were left to the latter, while the former managed the less life threatening situations. Telemonitoring had been thus complexified introducing a division of labour (and differentiated training courses) between the nurses themselves. Once again, the content of the innovation and its context showed to be tightly connected and mutually dependent.

\subsubsection{The many meanings of a non-ending}

This story has a non-conclusion. After almost four years from its inception, the procedure for costing and making telemonitoring a refundable service is still ongoing, as the pilot was severely delayed due in part the problems illustrated above. The necessary data were however collected and in view of the very positive results, the Lombardy region has recently publicly committed to formalise and operationalize the reimbursement of this procedure when used in alternative to the traditional approaches.

Interesting enough, this non conclusion is just as relevant, if not more relevant, than a nicer Hollywood style (happy) ending. The fact that the process is still ongoing can probably be attributed to the proverbial long-windedness of Italian policy making processes. However, the slowness and delay is also a sign that with the new translation telemonitoring has become a scientific and economic "fact" and has started to be perceived as a taken for granted procedure. The fact that the interest and the excitement has moved elsewhere is a sign that telemonitoring is starting to become institutionalised as a "normal" way of approaching CHF, that is to say, telemonitoring is starting to become simply medicine. From the perspective developed here, the disappearance from the headlines is a sign of success. As Sund and Rinde (1995) put it in one of the first articles on telemedicine, the success of this new approach will coincide with its demotion: "when the technique becomes successful...the term telemedicine will disappear" (Sund and Rinde, 1995, p. 24). This in turn, is an important step in the circulation of telemonitoring. To the extent that telemonitoring becomes simply a better and cheaper way of doing cardiology, it will become so prominent that it will be impossible to ignore. In other words, it will become a normative bandwagon (Fujimura, 1995) or a fashion (Czarniawska and Joerges, 1995; Czarniawska and Sevon, 1996): thanks to the combined force of law, scientific evidence, and funding, it will be those who try to ignore it, and those who fail to promptly implement it, that will have to justify their inaction. All the signs indicate that this is starting to happen at least in 
Europe and North America, where telemedicine is now both a growing market and a legitimate, albeit still new, medical specialty with its journals, conferences, and degrees.

There is, moreover, another interesting way in which this story has not yet ended. As often happens, the original project has generated a number of further initiatives. Two are worth mentioning here. One is the extension of the Lombardy pilot just described at national level. In many ways, this is a validation of the original strategy put in place by the people at G., who had foreseen that by conquering Lombardy that would have been in good position to expand their influence at national level. The second one, however, aims to extend telemonitoring to new specialities and new conditions. Telemonitoring is thus used for the care of patients with severe respiratory problems as well as for supporting the early discharge of patients after heart surgery. Telemonitoring CHF patients has thus been translated and metamorphosised into something else, a new idea that triggered a new wave of translation and a new process of circulation. Instead of the picture of a curve which ends, we are left with the image of a rhizome that has just sprung a new shoot from one of its nodes.

\section{DISCUSSION}

In the previous sections I used the toolkit of the Sociology of Translation for discussing the process through which tele-cardiology was established as a legitimate and accepted way of treating serious chronic heart failure in Italy.

The case study illustrates first that the circulation and spread of medical innovation requires the building of alliances between various actors including individuals, groups, institutions as well as machines. This effect must be obtained through a number of chains of translations based on the capacity of the innovation to serve a variety of different interests. Like a rhizome that extends itself by growing new branches and rooting itself in new ground, the innovation spreads far from its original place gaining force and momentum in the process. This, in turn, increases the importance of its promoters, who were literally riding the wave of change that they successfully put in motion and sustained.

Second, the case study also suggests that a set of orderly stages is hardly capable of capturing the reality of the process of medical and other types of innovations. In the case study, the spread of telemonitoring resembled more the successive waves of recruitment in a political campaign than a process of communication or the chemical process of dispersion fathomed by traditional diffusion studies. Most important, instead of the diffusion of a fixed entity that was implemented in different locales, we found the very object of translation changed along the process, as did all those involved.

Third, and strictly related to the former, the case study reveals the importance of stabilisation and closure efforts in the process of innovation in healthcare (and elsewhere), what May et al. (2003) called the normalisation of new ways of doing medicine. The case of telemonitoring suggests that the successful development and circulation of medical innovations depends on both forming a vast and increasingly complex network and on making this network stable and irreversible at local and at translocal level. At local level, this implied aligning heterogeneous actors and interests into a coherent whole, as well solving in practice the inevitable contradictions between the old ways of working and professional identities and the new tasks and division of labour performed by telemonitoring. The importance of this aspect comes fully to the 
fore if we compare the present case with the May and Ellis (2001) discussion of a failed attempt at introducing telemedicine in a UK region. In that case, the failure of forming an "alliance-into-network" (p.1000) around a protocol proved fatal for the implementation of telecare.

At global level, the normalisation implies the enrolment of increasingly more powerful allies which helped to define telemonitoring as an appropriate and legitimate way of delivering care. This too was achieved through the deployment of strategic intermediaries such as the protocol and the service model. As it appears from the case, stabilisation through accommodation, codification, and standardisation at both levels was a persistent concern of the lead characters in our story. Being very skilled doctorsociologists, they were well aware that medical innovations succeed only when these two levels are pulling in the same direction. We can see, then, that the networked model of the innovation process purported by the Sociology of Translation fits particularly well with the reality of healthcare, where normalization rather than adoption or diffusion is the issue (May et al., 2003). As in healthcare different means by which care or service can be delivered can co-exist side by side, evidence or information alone can never result automatically in the take up of innovations (Ferlie et al., 2005). Innovations have literally to grow from "somewhere" through the progressive establishment of connections between normalised local instances as described in the case study, until the network they form is large enough that it cannot be ignored -so that more local instances will get on board.

Finally, the case study showed that the circulation and institutionalisation of innovation is intrinsically a political process. Most of the activities illustrated in the case study constituted attempts at controlling and governing processes, conduct, and relationships in view of the promotion and support of identifiable configurations of interests, although "interest" was understood in a broad, and not necessarily economic sense. Power, in turn, was understood as a way of describing the outcome of the translation process. As we have seen, translating empowers those who are able to ride the wave of change. Doctor F., his firm, and the Centre of G. all derive their increasing visibility, prestige, and centrality from the position they manage to carve themselves in the association they contributed to build. Their power derived from, and was performed by, the relationship they had managed to assemble. In other words, the position they had achieved was clearly dependent on keeping those relationships in place: only to the extent that tele-cardiology could become a new obligatory point of passage for all those involved in caring for CHF patients, and only to the extent that they could remain the obligatory point of passage in this network could they maintain their position. Half way between Aladdin and Penelope, they had to keep re-weaving the magic carpet that allowed them to fly sky high.

This latter point is extremely consequential for the study of innovation. Most innovation stories have to do with entrepreneurship and network building: a person or a group of people encounter a new idea and using their capital (social, financial, intellectual, or other) they put in place the network and the conditions so that the idea can be turned into an innovation. Very often the network does not hold, and the innovation never comes to light. At other times, the network holds and we have both an innovation and entrepreneur to be interviewed. This way at looking at the innovation process, no matter how commonsensical it may appear, has two limitations which may hamper the effort of understanding what happened. First, by starting from human actors, it inherently 
obscures the role of non human entities in the process. Second, it predisposes us to believe the heroic narratives that entrepreneurs often use for describing themselves. Entrepreneurs will be necessarily tempted to narrate themselves in first person because this reinforces their role as spokesperson for the network that sustains them. In other words, the narrative told by entrepreneurs are often politically informed and aimed at reinforcing their position by reinterpreting their genealogy, not unlikely the powerful historical figures who hired poets who would celebrate their direct lineage from some divinity. Researchers who accept this narrative at face value fall for the fallacy that Bourdieu (1990) described as "complicitous" social research -they are unwittingly translated by the person they interview!

It follows that much is to be gained if we focus on the entrepreneurship process instead of on the entrepreneurship-persona (or the change agent or the innovation champion). One of the basic tenets of the sociology of translation is that we should not attribute agency before we start to investigate a phenomenon. Agency is necessarily a relational concept, that is, what counts as a source of agency depends on the specific net of actions. We should remain open to the possibility that the source of some events and activity is actually a non human actant. In our case study, for example, the innovation process depended just as much on the work of the senior cardiologist at G. and the doctor-entrepreneur as it did on the work performed by the (still) empty label "telemonitoring". When put in circulation, this label acted as catalyst and mediator turning a landscape of loosely coupled interests into a net of action (Czarniawska and Joerges, 1995), that is, it did some work at establishing relationships and making alliances possible. The idea/label of "telemonitoring" thus wove (or precipitated, if the idea of weaving sounds too anthropomorphic) a network of relationships and attracted interest and enrolments. Its role in the emergence of telemonitoring was thus bringing together a variety of interests and allowing a hypothetical set of interconnected elements to establish themselves as a unit which can act as one - constituting itself as an actornetwork. This, in turn, made both the position of the entrepreneur and of the champion possible, as each of these provided an impulse to the alignment process. Had we limited to focus on the skills and personal characteristics of the entrepreneurs or on the novelty of the idea, we would have not been able to understand the different aspects of the process and the roots of the success of telemedicine in northern Italy.

The study presented also provides some insights into the specific challenges posed by the introduction of telemedicine in general and telecare in particular. The case study corroborates the observation of other studies (see, e.g. Mort et al. 2003; Hanlon et al. 2005; Barlow et al, 2006; Nicolini, 2006; 2007) which suggest that the take up of telecare implies a modification of a variety of practices, material and institutional arrangements, and professional identities which had all to shift in order for telecare to become viable. In so doing, I substantiate the view put forward by Barlow et al (2006) that telecare exhibits a high level of structural and operational complexity which renders its introduction particularly challenging. As it clearly emerges in the study, while telecare promises to radically change our view of healthcare (see May et al, 2005; Finch et al, 2003; Finch et al, 2006 for a recent discussion) it also involves a large number of stakeholders, intersects different care processes, requires close integration between services, and shifts costs and benefits in an unequal way between different parts of the healthcare system (Barlow et al, 2006, p.398). The flip side of the benefits of telecare is thus the inherent difficulty in its introduction due to the necessity of producing an alignment between a variety of different, and often recalcitrant, aspects and conditions. 
Before concluding, it is also worth considering some of the limitations of the present research. For one thing, of course, the present study has all the limits of single case study. By design, in fact, this study - as well the approach it illustrates - favours depth over generality and hence cannot be used for developing a general rule-like statement. It must be added, however, that the translation approach alerts us that all innovations are necessarily 'local', and that the creation and maintenance of uniformities and general standards is something that needs to be explained empirically and not taken for granted. Phenomena such as the well known 'S-curve' are thus literally effects of a particular way of studying innovations - which of course doesn't make the phenomena less real or less objective and only invites us to develop alternatives ways of understanding it. Another limitation derives from the fact that the study mainly focuses on the early journey of telecare, describing what diffusion scholars would describe as its invention. It could be claimed, for example, that once standardised through the process described at the end of the case study, the telecare package will travel much quicker and according to processes similar to what is described by diffusion scholars. In this sense, as noted by Van de Ven and Hargrave (2003), the approach taken here is not necessarily incompatible with other accounts, and each could be used for explaining different phases of the life of an innovation. At the same time, one could argue that complex practices and heterogeneous arrangements such as telecare are very different from commodity-like innovations such as medicines and clinical instruments, so that different theories should be used for addressing the circulation of different types of innovations.

\section{CONCLUDING REMARKS}

My aim in this paper was to support the idea that the Sociology of Translation is a promising way for producing proximal descriptions of the circulation and stabilisation of medical innovation which brings fully to the fore its processual, networked, and interests lead nature. By applying the approach to the study of the emergence of telemonitoring in Northern Italy, I intended to show that this vocabulary and conceptual toolkit is particular apt at analysing and narrating innovation as an unending, uncertain distributed process through which ideas are translated in practice and coalition between humans, artefacts, and institutions are forged.

As it appeared in the case study, the conceptual vocabulary of the Sociology of Translation is especially fitting for producing processual and interpretive studies of healthcare innovation in that it foregrounds its networked, political, heterogeneous, and contingent nature. This approach particularly brings to the fore the networked nature of the innovation and the distributed and collective authorship of innovations.

A translation approach also underscores that politics and power are not an accessory or a barrier to the circulation of innovation and, on the contrary, they are inherent aspects of this phenomenon. Describing both the circulation and the mainstreaming of innovation is very much describing the process through which configurations of interests and concerns emerge and are anchored by specific social and material arrangements: as it promoted and supported very specific emerging configuration of interests over others, telecare was never politically neutral. Nor was the appeal to scientific evidence sufficient: in healthcare innovation, as in the broader scientific world, evidence is the result of political process, both the arena where the controversies are fought and the object which needs to be seized. Evidence is the name of a battle that has been won. 
Last, but certainly not least, the approach is inherently heterogeneous and contingent. For one thing, it emphasises that for in order to understand innovation we need to accept that technical, scientific, economic, and political considerations are inextricably bound into an organic whole. In this way, it downplays rigid dichotomies, as that between circulation and implementation, and between human and non-human elements. By acknowledging the performative power of non human actants, it thus helps us in developing narratives which acknowledge the distributed and complex nature of the innovation landscape and do not necessarily ascribe innovations to quasi heroic entrepreneurial actors. At the same time, by tracing the innovation process as it develops or not develops right from the start, it reminds us that the fate of innovation is highly contingent and uncertain, and that while things are the way they are, they could also have been very different.

\section{REFERENCES}

Barlow, J., S. Bayer and R. Curry (2003). 'The design of pilot Telecare projects and their. integration into mainstream service delivery', Journal of Telemedicine and Telecare, 9, pp. 1-3.

Barlow, J., S. Bayer and R. Curry (2006). 'Implementing complex innovations in fluid multi-stakeholder environments: Experiences of 'telecare', Technovation, 26, pp.396 406.

Bourdieu, P. (1990). The Logic of Practice. Cambridge, Polity Press (or. ed. 1980).

Callon, M., (1986). 'Some elements of a Sociology of Translation: Domestication of the scallops and fishermen of St. Brieuc Bay'. In Law, J. (ed) Power, action and belief: a new sociology of knowledge? pp. 196-233. London, Routledge,

Coleman, J. S., K. Elihu and M. Herbert (1966). Medical Innovation: A diffusion Study. New York, Bobbs-Merril.

Cooper, R. and J. Law (1995). 'Organization: Distal and Proximal View'. In S. Bacharach, P. Gagliardi, and B. Mundell (eds.), Research in the Sociology of Organizations. Studies of Organizations in the European Tradition, 13, pp. 237-274.

Czarniawska, B. and T. Hernes (eds.) (2005). Actor-Network Theory and Organizing. Copenhagen, Liber.

Czarniawska, B. and J. Joerges (1995). 'Winds of Organizational Change: How ideas Translate into objects and Actions'. In S., Bacharach, P., Gagliardi and B., Mundell (eds) Studies of the Organisations in the European Tradition. Research in the Sociology of Organizations, 13, pp. 171-210.

Czarniawska, B.and G. Sevón (eds) (1996). Translating Organisational Change. Berlin, de Gruyter.

Czarniawska, B.and G. Sevón (eds) (2005). Global ideas. How Ideas, Objects and Practices Travel in the Global Economy. Copenhagen, Liber.

Dopson, S. (2001). 'Applying an Eliasian Approach to Organizational Analysis', Organization, 8, pp.515-535 
Dopson, S. (2005). 'The Diffusion of Medical Innovations: Can Figurational Sociology Contribute?' Organization Studies, 6, pp. 1125-1144.

Dopson, S. and L. Fitzgerald (eds.) (2005). Knowledge in Action? The diffusion of innovations in health care. Oxford, University Press.

Fasol, R. and D. Nicolini (2004). 'Imprenditorialità nel campo della telemedicina'. In Silvia Gherardi e Antonio Strati (eds.), La telemedicina: tra tecnologia e organizzazione, pp.120-132. Roma, Carocci.

Ferlie, E., L. Fitzgerald, M. Wood and C. Hawkins (2005). 'The Nonspread of Innovations: The Mediating Role of Professionals', Academy of Management Journal, 48, pp.117-134.

Finch, T., May, C., Mort, M., Mair, F.and Mort, M. (2003). 'Integrating service development with evaluation in telehealthcare: an ethnographic study', British Medical Journal, 327, pp. 1205-1209.

Finch, T., C. May, M. Mort and F.Mair (2006). 'Telemedicine, telecare, and the future patient: innovation, risk and governance'. In A. Webster and S. Wyatt (eds.), New Technologies in Health Care: Challenge, Change and Innovation, pp. 84-96. New York, Palgrave Macmillan.

Fitzgerald, L., E. Ferlie, M. Wood and C. Hawkins (2002). 'Interlocking interactions, the diffusion of innovations in health care', Human Relations, 55, pp. 1429-1449

Fleuren, M., K. Wiefferink and T. Paulussen (2004). 'Determinants of Innovation within Health Care Organizations: Literature Review and Delphi Study', International Journal of Quality Health Care, 16, pp. 107-23.

Fujimura, J. H. (1995). 'Ecologies of Action: Recombining Genes, Molecularizing Cancer, and Transforming Biology'. In S. L. Star (ed.). Ecologies of Knowledge: Work and Politics in Science and Technology, pp. 302-346. Albany, NY, SUNY Press.

Geroski, P. A. (2000). 'Models of Technology Diffusion', Research Policy, 29, pp. 603625.

Greenhalgh T., G. Robert, P. Bate, O. Kyriakidou, F. Macfarlane and R. Peacock (2004 a). How to spread good ideas. A systematic review of the literature on diffusion, dissemination and sustainability of innovations in health service delivery and organisation. London: NCCSDO, available on line at: www.sdo.lshtm.ac.uk (Accessed 22 January 2007) .

Greenhalgh, T., R. Glenn, F. Macfarlane, P. Bate, and O. Kyriakidou (2004 b). 'Diffusion of Innovations in Service Organizations: Systematic Review and Recommendations', Milbank Quarterly, 82, pp. 581-629.

Hanlon, G., T. Strangleman, J. Goode, D. Luff, A. O'Cathain and D.Greatbatch (2005). 'Knowledge, technology and nursing: The case of NHS Direct', Human Relations, 58, pp. 147-171.

Hargrave, J. and A. Van de Ven (2006). 'A collective Action model of Institutional Innovation', Academy of Management Review, 31, pp. 864-888..

Latour, B. (1987). Science in Action. Milton Keynes, Open University. 
Latour, B. (2005). Reassembling the Social. An Introduction to Actor-Network-Theory. Oxford, University Press.

Law, J. (1986). 'On Power and Its Tactics: a View from the Sociology of Science', The Sociological Review, 34, pp. 1-38.

Law, J. (1992). 'Notes on the Theory of the Actor-Network: Ordering, Strategy, and Heterogeneity', System Practice, 5, pp. 379-393.

Lehoux P., C. Sicotte, J.-L. Denis, M. Berg and A. Lacroix (2002). 'The Theory of Use Behind Telemedicine: How Compatible With Physicians' Clinical Routines?' Social Science \& Medicine, 54, pp. 889-904.

May C. and N.T. Ellis (2001). 'When Protocols Fail: Technical Evaluation, Biomedical Knowledge, and the Social Production of 'Facts' about a Telemedicine Clinic', Social Science and Medicine, 53, pp. 989-1002

May, C., T. Finch, F. Mair and M. Mort, (2005). 'Towards a wireless patient: chronic illness, scarce care and technological innovation in the United Kingdom', Social Science \& Medicine, 61, pp.1485-94.

May, C., R. Harrison, T. Finch, A. MacFarlane, F. Mair and P.Wallace (2003). 'Understanding the normalization of telemedicine services through qualitative evaluation', Journal of American Medical Information Association, 10, pp.596-604.

McMurray, J. J. and S. Stewart (2003). 'The burden of heart failure', European Heart Journal Supplements, 4 , pp. D50-D58.

Mort, M., C. May and T. Williams (2003). 'Remote doctors and absent patients: acting at a distance in telemedicine', Science, Technology \& Human Values, 28, pp.274-95.

Nicolini, D. (2006). 'The work to make telemedicine work: A social and articulative view’, Social Science \& Medicine, 62, pp.2754-2767.

Nicolini, D. (2007). 'Stretching out and expanding medical practices. The case of telemedicine', Human Relations, 60, pp. 889-920.

Ricci, F. (2002). 'The Italian national telemedicine programme', Journal of Telemedicine and Telecare, $\mathbf{8}$, pp. 72-80.

Robertson, M. and J. Swan (1996). 'The Role of Networks in the Diffusion of Technological Innovation', Journal of Management Studies, 33, pp. 335- 361

Rogers, E. M. (1995). Diffusion of Innovation (4th ed.). New York, Free Press.

Rorty, R. (1989). Contingency, irony and solidarity. Cambridge, University Press.

Sackett, D., S. Strauss, W. Richardson, W. Rosenberg and R. Haynes, (2000). Evidence Based Medicine: how to practice and teach EBM. Toronto, Churchill and Livingstone.

Scalvini, S. and A. Giordano (2002). 'L'intervento Multidisciplinare Nei Pazienti Con Scompenso Cardiaco Cronico: Il Ruolo Della Telemedicina', Monaldi Archives for chest disease, 58,. pp. 252-255.

Star, S. L. and J. R. Griesemer (1989). 'Institutional ecology, 'translations' and boundary objects: amateurs and professionals in Berkeley's Museum of Vertebrate Zoology, 1907-39', Social Studies of Science, 19, pp. 387-420. 
Sund, T. and E. Rinde (1995). 'Telemedicine: Still waiting for users', Lancet, 346 (suppl). pp. s24.

Swan, J. and H. Scarbrough (2005). 'The politics of networked innovation', Human Relations, 58, pp. 913-943.

Swan, J. and S. Newell (1995). 'The role of professional associations in technology diffusion', Organization Studies, 16, pp. 847-874.

Van de Ven, A. H. and T. Hargrave (2003). 'Social, Technical, and Institutional Change: A Literature Review and Synthesis'. In M. S. Poole and A. H. Van de Ven (Eds.) Handbook of Organizational Change, pp. 259-303. Oxford, University Press,.

Van de Ven, A. H., D. Polley, R.Garud and S.Ventakaraman (1999). The Innovation Journey. Oxford, University Press.

Van de Ven, A. and E. Rogers (1988). 'Innovation and Organisations: Critical perspectives', Communication Research, 15, pp. 632-651. 